\title{
Stability of non-autonomous difference equations: simple ideas leading to useful results
}

\author{
Eduardo Liz* \\ Departamento de Matemática Aplicada II, E.T.S.E. Telecomunicación, Universidade de Vigo, \\ Campus Marcosende, 36310 Vigo, Spain
}

(Received 2 March 2009; in revised form 20 May 2009)

We address the stability properties in a non-autonomous difference equation

$$
x_{n+1}=f\left(n, x_{n}, \ldots, x_{n-k}\right), \quad n \geq 0,
$$

where $f$ is continuous, and the zero solution is assumed to be the unique equilibrium. We focus our discussion on two techniques motivated by stability results for functional differential equations (FDEs) that proved recently to be useful in the frame of difference equations too. The first one involves the use of discrete inequalities and monotonicity arguments, and it is inspired by the so-called Halanay inequality; the second one is based on the well-known 3/2 stability results for FDEs. We give further insight into the simple ideas that are behind these methods, prove some new results and show applications and open problems.

Keywords: difference equations; exponential stability; asymptotic stability; discrete Halanay-type inequalities; monotone maps

AMS Subject Classification: 39A10; 39A11; 47H07

\section{Introduction}

Difference equations are the appropriate mathematical representation for discrete processes, which have special importance in areas such as population dynamics and economics (see the monographs $[1,10,11,15,24,48])$. Recently, the issue of global stability has attracted special attention in the areas indicated above, and in others such as social sciences [48], numerical analysis [3] and neural networks [42,46,56].

The simplest case is given by the first-order difference equation

$$
x_{n+1}=f\left(x_{n}\right), \quad n \geq 0,
$$

which is widely investigated in the literature. Difference equations of order greater than one are much less studied, and they have great importance in applications where the state (for example, the size of a population) after $n$ steps depends on the previous $k+1$ states $(k \geq 1)$. We consider a general nonlinear difference equation of order $k+1$

$$
x_{n+1}=f_{n}\left(x_{n}, \ldots, x_{n-k}\right), \quad n \geq 0,
$$

where $f_{n}: \mathbb{R}^{k+1} \rightarrow \mathbb{R}$ is a continuous function for each integer $n \geq 0$. Note that equation (1.1) is in general non-autonomous. As it is well known, a solution of (1.1) with initial data

\footnotetext{
*Email: eliz@dma.uvigo.es 
$\left(\alpha_{0}, \ldots, \alpha_{-k}\right)$ is a real sequence $\left\{x_{n}\right\}_{n \geq-k}$ that satisfies equation (1.1) for $n \geq 0$, and $x_{i}=\alpha_{i}$ for $i=-k, \ldots, 0$. We assume that the zero solution is the unique equilibrium, i.e. $f_{n}(c, \ldots c)=c$ for some real constant $c$ and all $n \in \mathbb{N}$ if and only if $c=0$. We note that in many applications there is a positive equilibrium, but the model can be written in this form after a change of variables.

The main aim of this paper is to provide a brief overview of several recent results for the stability of the equilibrium in equation (1.1). We give further insight into the simple ideas that are behind these methods, state new results, show some applications, discuss some related conjectures and formulate new open problems.

We deal with two techniques that have two facts in common: first, they are inspired by the stability results for functional differential equations (FDEs); and, second, they involve at some extent the max functional.

Section 2 is devoted to the first method, based on the so-called Halanay-type inequalities, while the second method is treated in Section 3; it is inspired by the famous 3/2 stability results for FDEs developed by many authors such as Myshkis, Yorke, Yoneyama and Krisztin, among others. For more discussions and the relationship between these two topics, see [20].

First, we recall some notations and definitions that will be used in the paper. For a vector $\boldsymbol{x}=\left(x_{0}, \ldots, x_{k}\right) \in \mathbb{R}^{k+1}$, we denote $\|\boldsymbol{x}\|_{0}=\max \left\{\left|x_{0}\right|, \ldots,\left|x_{k}\right|\right\}$.

DeFINITION 1.1. For equation (1.1), we understand that

- the zero solution is stable if for every $\varepsilon>0$ and $n_{0}>0$ there exists $\delta\left(\varepsilon, n_{0}\right)>0$ such that if $\left\{x_{n}\right\}$ is a solution of (1.1) with $\left\|\left(x_{n_{0}-k}, \ldots, x_{n_{0}}\right)\right\|_{0} \leq \delta$, then $\left|x_{n}\right|<\varepsilon$ for all $n \geq n_{0}$;

- the zero solution is uniformly stable if for every $\varepsilon>0$ the number $\delta$ in the previous definition may be chosen independent of $n_{0}$;

- the zero solution is attracting if there exists $\mu>0$ such that $\lim _{n \rightarrow \infty} x_{n}=0$ for any solution $\left\{x_{n}\right\}$ of (1.1) such that $\left\|\left(x_{0}, \ldots, x_{-k}\right)\right\|_{0} \leq \mu$;

- the zero solution is uniformly asymptotically stable if it is attracting and uniformly stable;

- the zero solution is globally attracting if $\lim _{n \rightarrow \infty} x_{n}=0$, for all solutions $\left\{x_{n}\right\}$ of (1.1);

- the zero solution is globally asymptotically stable (or simply globally stable) if it is stable and globally attracting and

- the zero solution is globally exponentially stable if there exist constants $M>0$ and $\lambda \in(0,1)$ such that, for every solution $\left\{x_{n}\right\}_{n \geq-k}$ of $(1.1)$, the inequality

$$
\left|x_{n}\right| \leq M \lambda^{n}\left(\max _{-k \leq i \leq 0}\left\{\left|x_{i}\right|\right\}\right)
$$

holds for all $n \geq 0$.

We note that analogous definitions can be stated in the frame of difference equations in a Banach space $X$, replacing everywhere the modulus of a real number by the norm of a vector in $X$.

\section{Halanay-type results}

In this section, we present some sufficient conditions for the global stability of the equilibrium often referred to as Halanay-type results. They are inspired by the elegant 
ideas developed by Halanay [19] to prove a stability result for delay differential equations based on differential inequalities involving the max functional. As far as we know, the first results for difference equations in this direction may be found in [4] and [41], where they are presented in the frame of numerical approximations of FDEs. This makes the statements of the related results (Theorem 7.1 in [4] and Theorem 3.1 in [41]) quite complicated, and the simple ideas of Halanay are somehow hidden in the proofs.

We put a special emphasis on these ideas, showing how to generalize the known results in different ways, including their application to the stability of difference equations in Banach spaces. We remark that all results in this section establish sufficient conditions for the global exponential stability.

To begin our discussion, we state a result from [34].

Theorem 2.1 [34, TheOrem 2]. Assume that $0 \leq a<1$ and there exists a positive constant $b$ such that

$$
\left|f_{n}(\boldsymbol{x})\right| \leq b\|\boldsymbol{x}\|_{0}, \quad \forall \boldsymbol{x} \in \mathbb{R}^{k+1} \quad \text { and } \quad n \geq 0 .
$$

If $0<b<1-a$, then there exists a constant $\lambda_{0} \in(0,1)$ such that

$$
\left|x_{n}\right| \leq\left(\max _{-k \leq i \leq 0}\left\{\left|x_{i}\right|\right\}\right) \lambda_{0}^{n}, \quad n \geq 0
$$

for every solution $\left\{x_{n}\right\}$ of equation

$$
x_{n+1}=a x_{n}+f_{n}\left(x_{n}, \ldots, x_{n-k}\right), \quad n \geq 0,
$$

where $\lambda_{0}$ can be chosen as the unique root in the interval $(0,1)$ of equation

$$
\lambda^{k+1}-a \lambda^{k}-b=0 .
$$

As a consequence, the zero solution of equation (2.3) is globally exponentially stable. Obviously, equation (1.1) can be written as (2.3) with $a=0$. Hence, we have the following corollary.

COROLlary 2.2. The zero solution of equation (1.1) is globally exponentially stable if (2.1) holds for some $b \in(0,1)$.

We note that, in this case, we can choose $\lambda_{0}=b^{1 /(k+1)}$ in (2.2).

Remark 1. Theorem 3.1 in [41] is a direct consequence of Corollary 2.2.

Remark 2. Corollary 2.2 may also be proved by a simple induction argument (see [6], Theorem 2.1), and it also applies to equation (2.3) since, under condition (2.1),

$$
\left|a x_{0}+f_{n}(\boldsymbol{x})\right| \leq(a+b)\|\boldsymbol{x}\|_{0}, \quad \forall \boldsymbol{x}=\left(x_{0}, \ldots, x_{k}\right) \in \mathbb{R}^{k+1} \text { and } n \geq 0,
$$

with $a+b<1$. Thus, we recover Theorem 2.1 from Corollary 2.2.

It should be noted that an autonomous version of this corollary was proved by Sedaghat in [47, Corollary 2] (see also Section 4.3 in the monograph [48] of the same 
author). Memarbashi [39,40] recently extended Sedaghat's arguments to get similar stability results for the non-autonomous difference equation (1.1).

We briefly discuss the relation between our approach and Sedaghat's results in more detail. In our opinion, the real difference is that, while Sedaghat's result relies on a 'contraction argument', the Halanay-type approach is based on monotonicity arguments for positively homogeneous operators (like the max functional).

Remark 3. An interesting approach to contraction-like criteria for studying the asymptotic behaviour of non-autonomous difference equations can be found in the recent paper of Ey and Pötzsche [14].

The first advantage of the Halanay-type approach for equation (2.3) is the following: one can check that, for $a>0$, the constant $\lambda_{0}$ given by Theorem 2.1 is less than $(a+b)^{1 /(k+1)}$, and hence Theorem 2.1 provides a sharper decaying rate.

However, a more interesting aspect is that the same ideas used in [34] may be easily extended to get new stability results.

We recall the definition of a monotone function. Given two Banach spaces $X, Y$ partially ordered by cones $X_{+}, Y_{+}$, respectively, a map $F: X_{+} \rightarrow Y_{+}$is monotone if $F(x) \leq F(y)$ for all $x, y \in X_{+}$such that $x \leq y$.

The usual pointwise ordering in $\mathbb{R}^{k+1}$ is induced by the cone

$$
\mathbb{R}_{+}^{k+1}=\left\{\left(x_{0}, x_{1}, \ldots, x_{k}\right) \in \mathbb{R}^{k+1}: x_{i} \geq 0, \forall i=0,1, \ldots, k\right\} .
$$

If $h: \mathbb{R}_{+}^{k+1} \rightarrow \mathbb{R}_{+}$is monotone, then the autonomous difference equation

$$
x_{n+1}=h\left(x_{n}, \ldots, x_{n-k}\right), \quad n \geq 0
$$

is monotone in the sense that if $\left\{x_{n}\right\},\left\{y_{n}\right\}$ are two solutions of (2.5) such that $\left(x_{0}, \ldots, x_{-k}\right) \leq\left(y_{0}, \ldots, y_{-k}\right)$, then $x_{n} \leq y_{n}$ for all $n \geq 0$.

Associated to (2.5), we can define a map $T_{h}: \mathbb{R}_{+}^{k+1} \rightarrow \mathbb{R}_{+}^{k+1}$ by

$$
T_{h}\left(x_{0}, \ldots, x_{k}\right)=\left(h\left(x_{0}, \ldots, x_{k}\right), x_{1}, \ldots, x_{k-1}\right) .
$$

It is clear that equation (2.5) is monotone if and only if $T_{h}$ is monotone. This fact allows us to employ some abstract results for monotone operators in cones due to Krause [25], and Kloeden and Rubinov [23].

The proof of Theorem 2.1 is based on a comparison result (Theorem 1 in [34]), which is demonstrated using two basic facts:

(1) The difference equation

$$
x_{n+1}=a x_{n}+b \max \left\{x_{n}, \ldots, x_{n-k}\right\}, \quad n \geq 0
$$

is monotone with respect to the usual pointwise ordering in $\mathbb{R}^{k+1}$ if $a, b \geq 0$.

(2) Equation (2.4) has a unique real root $\lambda_{0} \in(0,1)$ since $b>0, a+b<1$.

In order to generalize Theorem 2.1, we state a new comparison result that is proved following the same arguments mentioned above in a more general setting.

We recall that a map $h: \mathbb{R}_{+}^{k+1} \rightarrow \mathbb{R}_{+}$is positively homogeneous if $h(\lambda x)=\lambda h(x)$ for all $\lambda \geq 0$ and $x \in \mathbb{R}_{+}^{k+1}$. 
THEOREM 2.3. Assume that $h: \mathbb{R}_{+}^{k+1} \rightarrow \mathbb{R}_{+}$is continuous, monotone and positively homogeneous. Let the following properties hold:

(1) $\mathrm{h}(1,1, \ldots, 1)<1$

(2) Either $h(0,0, \ldots, 0,1)>0$ or $\lim _{\lambda \rightarrow 0^{+}} h\left(1, \lambda^{-1}, \ldots, \lambda^{-k}\right)>0$.

If $\left\{x_{n}\right\}_{n \geq-k}$ is a sequence of non-negative real numbers satisfying the inequality

$$
x_{n+1} \leq h\left(x_{n}, x_{n-1}, \ldots, x_{n-k}\right), \quad n \geq 0,
$$

then there exists a constant $\lambda_{0} \in(0,1)$ such that

$$
x_{n} \leq \max \left\{x_{0}, x_{-1}, \ldots x_{-k}\right\} \lambda_{0}^{n}, n \geq 0 .
$$

Moreover, $\lambda_{0}$ can be chosen as the unique root in the interval $(0,1)$ of equation

$$
\lambda^{k+1}=h\left(\lambda^{k}, \lambda^{k-1}, \ldots, \lambda, 1\right) .
$$

Proof. Let $\left\{y_{n}\right\}$ be a solution of the difference equation

$$
y_{n+1}=h\left(y_{n}, y_{n-1}, \ldots, y_{n-k}\right), n \geq 0 .
$$

Since $h$ is monotone, it is easy to prove by induction that if $\left\{x_{n}\right\}$ satisfies the inequality (2.7) and $x_{n} \leq y_{n}$ for $n=-k, \ldots, 0$, then $x_{n} \leq y_{n}$ for all $n \geq 0$.

Since $h$ is positively homogeneous, for $K>0$ and $\lambda>0$, the sequence $\left\{y_{n}\right\}$ defined by $y_{n}=K \lambda^{n}$ is a solution of equation (2.9) if and only if $\lambda$ is a solution of (2.8).

Assume first that $h(0,0, \ldots, 0,1)>0$. Define $F(\lambda)=\lambda^{k+1}-h\left(\lambda^{k}, \lambda^{k-1}, \ldots, \lambda, 1\right) . F$ is continuous on $[0,1], F(0)=-h(0,0, \ldots, 0,1)<0$, and $F(1)=1-h(1,1, \ldots, 1)>0$. Hence, there exists $\lambda_{0} \in(0,1)$ such that $F\left(\lambda_{0}\right)=0$. Moreover, an application of Theorem 4 in [25] proves that $\lambda_{0}$ is the unique non-negative solution of (2.8).

Thus, $\left\{y_{n}\right\}=\left\{K \lambda_{0}^{n}\right\}$ is a solution of (2.9) for every $K>0$. Choosing $K=\max \left\{x_{0}, x_{-1}, \ldots x_{-k}\right\}$, it is clear that $y_{n} \geq x_{n}$ for all $n=-k, \ldots, 0$. Hence, using the first part of the proof, we can conclude that $x_{n} \leq y_{n}=K \lambda_{0}^{n}$ for all $n \geq 0$.

If $h(0,0, \ldots, 0,1)=0$ but $\lim _{\lambda \rightarrow 0^{+}} h\left(1, \lambda^{-1}, \ldots, \lambda^{-k}\right)>0$, then the same arguments work, taking into account that, for $\lambda>0$, the equation (2.8) is equivalent to

$$
\lambda=h\left(1, \lambda^{-1}, \ldots, \lambda^{-k}\right) .
$$

In this case, the uniqueness of $\lambda_{0}$ follows from the application of [23, Corollary 3.1] to the operator $T_{h}: \mathbb{R}_{+}^{k+1} \rightarrow \mathbb{R}_{+}^{k+1}$ defined by (2.6).

Remark 4. The original Halanay-type result given in [34, Theorem 1] is a particular case of Theorem 2.3, with

$$
h\left(x_{0}, \ldots, x_{k}\right)=(1-a) x_{0}+b \max \left\{x_{0}, \ldots, x_{k}\right\} .
$$

It is clear that $h$ is continuous, monotone and positively homogeneous if $b>0$ and $a \leq 1$. Conditions (a) and (b) hold when $0<b<a$. For $a=1$ in (2.11), the same result was previously proved by Stević [49], and was applied to investigate the asymptotic behaviour of the solutions in an ecological model. 
We also derive from Theorem 2.3 some recent results that employ different functionals. For example, taking $h\left(x_{0}, \ldots, x_{k}\right)=(1-p) x_{0}+\prod_{i=0}^{r} \beta_{i} x_{h_{i}}^{\alpha_{i}}$, where $p, \alpha_{i}, \beta_{i}$ are positive real numbers, $0=h_{0}<h_{1}<\cdots<h_{r}=k, \sum_{i=0}^{r} \alpha_{i}=1, \prod_{i=0}^{r} \beta_{i}<p \leq 1$, we get Theorem 2.2 in [53]. Note that in this case $h(0,0, \ldots, 0,1)=0$, but $\lim _{\lambda \rightarrow 0^{+}} h$ $\left(1, \lambda^{-1}, \ldots, \lambda^{-k}\right)=+\infty$.

Other straightforward consequences of Theorem 2.3 are Theorem 2.2 in [2], Theorem 16 in [3] and Theorem 2.1 in [53].

Once, one has the comparison result for inequality (2.7), it is easy to prove a global stability result for non-autonomous difference equations, when the nonlinear part is dominated in some sense for the monotone positively homogeneous function $h$. For it, we use the variation of constants formula for (2.3)

$$
x_{n}=a^{n} x_{0}+\sum_{i=0}^{n-1} a^{n-i-1} f_{i}\left(x_{i}, \ldots, x_{i-k}\right), \quad n \geq 0,
$$

together with an idea taken from Halanay's book [19]; see the proof of [34, Theorem 2], where $h\left(x_{0}, \ldots, x_{k}\right)=\max \left\{x_{0}, \ldots, x_{k}\right\}$. Following the same arguments, but considering a general $h$ in the conditions of Theorem 2.3, we get the following generalization of Theorem 2.1.

THEOREM 2.4. Assume that $0 \leq a<1$ and there exists a continuous, monotone and positively homogeneous function $h: \mathbb{R}_{+}^{k+1} \rightarrow \mathbb{R}_{+}$such that

$$
\left|f_{n}\left(x_{0}, \ldots, x_{k}\right)\right| \leq h\left(\left|x_{0}\right|, \ldots,\left|x_{k}\right|\right), \forall\left(x_{0}, \ldots, x_{k}\right) \in \mathbb{R}^{k+1} \text { and } n \geq 0 \text {. }
$$

If $h(1,1, \ldots, 1)<1-a$, and either $h(0,0, \ldots, 0,1)>0$ or $\lim h\left(1, \lambda^{-1}, \ldots\right.$, $\left.\lambda^{-k}\right)>-a$, then equation (2.3) is globally exponentially stable. More precisely, there exists a constant $\lambda_{0} \in(0,1)$ such that $(2.2)$ holds for every solution $\left\{x_{n}\right\}$ of $(2.3)$, where $\lambda_{0}$ can be chosen as the unique root in the interval $(0,1)$ of equation

$$
\lambda^{k+1}-a \lambda^{k}=h\left(\lambda^{k}, \lambda^{k-1}, \ldots, \lambda, 1\right) .
$$

When $a=0$, this result is easily generalized to non-autonomous difference equations in Banach spaces. Let $X$ be a Banach space and consider the difference equation

$$
x_{n+1}=f_{n}\left(x_{n}, \ldots, x_{n-k}\right), \quad n \geq 0,
$$

with initial conditions $x_{-k}, \ldots, x_{0} \in X$, where $f_{n}: X^{k+1} \rightarrow X$ is continuous for every $n \geq 0$.

THEOREM 2.5. Assume that there exists a function $h: \mathbb{R}_{+}^{k+1} \rightarrow \mathbb{R}_{+}$in the conditions of Theorem 2 such that

$$
\left\|f_{n}\left(x_{0}, \ldots, x_{k}\right)\right\| \leq h\left(\left\|x_{0}\right\|, \ldots,\left\|x_{k}\right\|\right), \forall\left(x_{0}, \ldots, x_{k}\right) \in X^{k+1} \text { and } n \geq 0 .
$$

Then, equation (2.15) is globally exponentially stable. 
Proof. Let $\left\{x_{n}\right\}$ be a solution of equation (2.15). Using (2.16), we obtain

$$
\left\|x_{n+1}\right\|=\left\|f_{n}\left(x_{n}, \ldots, x_{n-k}\right)\right\| \leq h\left(\left\|x_{n}\right\|, \ldots,\left\|x_{n-k}\right\|\right), \quad n \geq 0 .
$$

Denote $v_{n}=\left\|x_{n}\right\|$ for $n=-r, \ldots, 0$, and $v_{n+1}=h\left(\left\|x_{n}\right\|, \ldots,\left\|x_{n-k}\right\|\right)$ for $n \geq 0$. Since $\left\|x_{n}\right\| \leq v_{n}$ for all $n \geq-k$, and $h$ is monotone, we have

$$
v_{n+1}=h\left(\left\|x_{n}\right\|, \ldots,\left\|x_{n-k}\right\|\right) \leq h\left(v_{n}, \ldots, v_{n-k}\right), n>0 .
$$

Theorem 2.3 ensures that

$$
\left\|x_{n}\right\| \leq v_{n} \leq \max \left\{v_{0}, \ldots v_{-k}\right\} \lambda_{0}^{n}=\max \left\{\left\|x_{0}\right\|, \ldots\left\|x_{-k}\right\|\right\} \lambda_{0}^{n}, \quad n \geq 0 .
$$

Moreover, $\lambda_{0}$ can be chosen as the unique root in the interval $(0,1)$ of equation $(2.8)$.

Remark 5. In the particular case when $h\left(x_{0}, \ldots, x_{k}\right)=\alpha \prod_{i=0}^{k} x_{i}^{p_{i}}$, where $\alpha>0, p_{i} \geq 0$ for $i=0,1, \ldots, k$, and $\sum_{i=0}^{k} p_{i}=1$, Theorem 2.3 states that equation (2.15) is globally exponentially stable if $\alpha<1$ and

$$
\left\|f_{n}\left(x_{0}, \ldots, x_{k}\right)\right\| \leq \alpha \prod_{i=0}^{k}\left\|x_{i}\right\|^{p_{i}}, \forall\left(x_{0}, \ldots, x_{k}\right) \in X^{k+1} \text { and } n \geq 0 .
$$

This result improves Theorem 3.1 (ii) in [5], where only the asymptotic stability is proved under the same conditions.

Theorem 2.5 allows us to get results of global exponential stability for systems of difference equations too.

As an example, let us consider a linear system of non-autonomous difference equations in $\mathbb{R}^{m}$

$$
u_{n+1}=\sum_{i=0}^{k} A_{i}(n) u_{n-k}, \quad n \geq 0,
$$

where $u_{n} \in \mathbb{R}^{m}$ and $A_{i}(n) \in \mathbb{R}^{m \times m}$, for all $n \geq 0$. We consider a norm in $\mathbb{R}^{m \times m}$ consistent with a vector norm in $\mathbb{R}^{m}$. The following result follows easily from Theorem 2.5.

Corollary 2.6. Assume that

$$
\sup _{n \geq 0} \sum_{i=0}^{k}\left\|A_{i}(n)\right\|=\alpha<1 .
$$

Then, system (2.17) is globally exponentially stable.

Proof. Let us denote $f_{n}\left(u_{0}, \ldots, u_{k}\right)=\sum_{i=0}^{k} A_{i}(n) u_{k}$, and take $h\left(x_{0}, \ldots, x_{k}\right)=\alpha \max$ $\left\{x_{0}, \ldots, x_{k}\right\}$. Then, (2.17) takes the form (2.15), and

$$
\left\|f_{n}\left(u_{0}, \ldots, u_{k}\right)\right\|=\left\|\sum_{i=0}^{k} A_{i}(n) u_{k}\right\| \leq \alpha \max \left\{\left\|u_{0}\right\|, \ldots,\left\|u_{k}\right\|\right\}=h\left(\left\|u_{0}\right\|, \ldots,\left\|u_{k}\right\|\right),
$$

for all $\left(u_{0}, \ldots, u_{k}\right) \in \mathbb{R}^{m(k+1)}$ and $n \geq 0$. An application of Theorem 2.5 shows that system (2.17) is exponentially stable.

An autonomous version of Corollary 2.6 was proved by Kipnis and Komissarova [21]. 
A special feature of the stability results included in this section is that they are very easy to apply. For example, another immediate application of Theorem 2.5 is Theorem 2.9 in [57], which provides the global exponential stability of a system of discrete-time Hopfield neural networks. For related results in the same area using Halanay-type theorems see [46]. Other fields of application are stability theory for models in economic dynamics, see $[6,12,28]$, and control theory [30].

On the other hand, the simple ideas used in the proofs of Theorems 1 and 2 in [34] led us to obtain other results in equations, where Theorem 2.4 does not apply. As an example, consider the non-autonomous difference equation

$$
x_{n+1}-x_{n}+p x_{n-m}+f_{n}\left(x_{n}, \ldots, x_{n-k}\right)=0, \quad n \geq 0,
$$

where $p>0$ is a real number, and $m, k$ are integers, where $1 \leq m \leq k$.

Assuming that (2.1) holds for some $b>0$, we cannot apply any of the previous results to get the exponential stability of (2.18). The main reason is that, contrary to what happens in the case $m=0, p<1$, the linear equation

$$
x_{n+1}-x_{n}+p x_{n-m}=0
$$

is not monotone with respect to the usual ordering in $\mathbb{R}^{k+1}$ for any value of $p>0$. However, we can find a different ordering for which it is monotone if an additional condition is assumed.

Thus, we can expect to get sufficient conditions for the exponential stability of (2.18), if we are able to use the same ideas involved in the proofs of the discrete Halanay-type results using different orderings in $\mathbb{R}^{k+1}$. This approach was developed in [36], using the discrete exponential ordering introduced in [26]. We note that, in this case, the generalized form of the variation of constants formula in terms of the fundamental solution is needed instead of (2.12) (see [36], for details).

The following corollary of Theorem 1.4 in [36] applies to equation (2.18).

Proposition 2.7. Assume that (2.1) holds for some constant $\mathrm{b}$ such that

$$
0<b<p \leq \frac{m^{m}}{(m+1)^{m+1}}
$$

Then, there exist two constants $\mathrm{M}>0$ and $\lambda_{0} \in(0,1)$ such that

$$
\left|x_{n}\right| \leq M\left(\max _{-k \leq i \leq 0}\left\{\left|x_{i}\right|\right\}\right) \lambda_{0}^{n}, \quad n \geq 0,
$$

for every solution $\left\{x_{n}\right\}$ of equation (2.18), where $\lambda_{0}$ can be chosen as the unique root in the interval $(m /(m+1), 1)$ of equation

$$
\lambda^{k+1}-\lambda^{k}+p \lambda^{k-m}-b=0 .
$$

Therefore, the zero solution of equation (2.18) is globally exponentially stable. 
Another way to get new stability results using the approach in this section consists in considering equation

$$
x_{n+1}-x_{n}=-\sum_{i=0}^{k} a_{i}(n) x_{n-i}+f_{n}\left(x_{n}, \ldots, x_{n-k}\right), \quad n \geq 0
$$

(where the non-autonomous part consists of a linear term and a nonlinear one), and manipulate it to get a new expression to which we can apply the Halanay-type stability results. Three different ideas in this direction were introduced in [35], [31] (see also [6]) and, more recently, in El-Morshedy's paper [12].

The following corollary of [31, Theorem 2.4] provides a new sufficient condition for the exponential stability in Equation (2.18).

Proposition 2.8. Assume that $\mathrm{mp}<1$ and (2.1) holds for some constant $\mathrm{b}$ such that

$$
0<b<p \frac{1-m p}{1+m p} .
$$

Then, equation (2.18) is globally exponentially stable.

We can apply [35, Theorem 4] to get a new condition that ensures the exponential stability of the zero solution of (2.18) by requiring, instead of (2.1), the following stronger condition on the nonlinearity $f$ :

$$
\alpha \min \left\{x_{0}, \ldots, x_{k}\right\} \leq f_{n}\left(x_{0}, \ldots, x_{k}\right) \leq \alpha \max \left\{x_{0}, \ldots, x_{k}\right\}, \quad \forall n \geq 0,
$$

for some positive constant $\alpha$. This is stated in the following result.

Proposition 2.9. Assume that condition (2.24) is satisfied for some constant $\alpha$ such that

$$
p+\alpha<\frac{1}{k}
$$

Then, equation (2.18) is globally exponentially stable.

Remark 6. Condition (2.24) is the discrete version of a similar assumption used in stability results for FDEs; in Section 3, we will come back to this type of 'min-max' hypotheses.

We emphasize that the stability conditions given for equation (2.18) in Propositions 2.7-2.9 are not comparable, and all of them follow from different generalizations of the Halanay-type Theorem 2.1.

\section{Stability results of 3/2-type and the use of EPCA}

In this section, we state some stability results using another approach that has also been motivated by the qualitative theory of FDEs, more precisely, by the so-called 3/2 stability results. In his famous paper [55], Yorke investigated the asymptotic stability of a nonlinear non-autonomous FDE

$$
x^{\prime}(t)=-F\left(t, x_{t}\right), \quad t \geq 0,
$$


where $F:[0, \infty) \times C \rightarrow \mathbb{R}$ is continuous, $C$ is the Banach space of the continuous real functions defined in $[-\tau, 0]$ and, for each $t \geq 0, x_{t} \in C$ is defined as $x_{t}(s)=x(t+s), \forall s \in$ $[-\tau, 0]$. A key assumption is a growth condition on $F$, namely, there is a constant $\alpha>0$ such that

$$
\alpha \min \left\{0, \min _{s \in[-\tau, 0]} \phi(s)\right\} \leq F(t, \phi) \leq \alpha \max \left\{0, \max _{s \in[-\tau, 0]} \phi(s)\right\}, \quad \forall \phi \in C .
$$

A generalization of (3.2) replacing constant $\alpha$ by $a(t)$, where $a: \mathbb{R}_{+} \rightarrow \mathbb{R}_{+}$is a nonnegative continuous function, was introduced by Yoneyama [54].

Some interesting variants of (3.2), involving the Riemann-Stieltjes integral, were introduced by Krisztin in [27]. One example is

$$
\sum_{i=0}^{k} \alpha_{i} \min _{s \in\left[-\tau_{i}, 0\right]} \phi(s) \leq F(t, \phi) \leq \sum_{i=0}^{k} \alpha_{i} \max _{s \in\left[-\tau_{i}, 0\right]} \phi(s), \quad \forall \phi \in C,
$$

where $0 \leq \tau_{0}<\cdots<\tau_{k}=\tau, \alpha_{0}, \ldots, \alpha_{k}$ are non-negative numbers, where $\sum_{i=0}^{k} \alpha_{i}>0$.

Note that condition (2.24) introduced in the previous section is a discrete version of (3.3), with $\alpha_{i}=0$ for $0 \leq i \leq k-1, \alpha_{k}=\alpha>0$.

The stability theorems obtained under the conditions mentioned above are called the $3 / 2$ stability results, because they require a restriction on the size of the delay which involves the number $3 / 2$. For example, in the main result of [55], it is required that $\alpha \tau<3 / 2$ to ensure the asymptotic stability of the zero solution in (3.1).

Condition (3.2) was generalized in [37] to extend Yorke's Theorem to a broader family of FDEs of the form (3.1), with applications in population dynamics and other areas.

Let $\beta$ be a non-negative real number, and define the rational function $R(x)=$ $x /(1+\beta x)$ for $x>-1 / \beta$. We call the following property the generalized Yorke condition:

$$
\alpha R\left(\min \left\{0, \min _{s \in[-\tau, 0]} \phi(s)\right\}\right) \leq F(t, \phi) \leq \alpha R\left(\max \left\{0, \max _{s \in[-\tau, 0]} \phi(s)\right\}\right),
$$

where the second inequality holds for all $\phi \in C$, and the first one for all $\phi$ such that $\min _{s \in[-1,0]} \phi(s)>-\beta^{-1} \in[-\infty, 0)$.

Note that for $\beta=0$, we recover the Yorke condition (3.2).

An easy way to get $3 / 2$ stability theorems for difference equations consists in exploiting the fact that a solution $\left\{x_{n}\right\}$ of the difference equation

$$
x_{n+1}-x_{n}+f_{n}\left(x_{n}, \ldots, x_{n-k}\right)=0, \quad n \geq 0,
$$

can be considered as the solution (at the integers) of a delay differential equation with piecewise constant arguments (EPCA), namely

$$
x^{\prime}(t)=-f([t], x([t]), \ldots, x([t-k])), \quad t \geq 0,
$$

where $f\left(n, x_{n}, \ldots, x_{n-k}\right)=f_{n}\left(x_{n}, \ldots, x_{n-k}\right)$, and $[z]$ denotes the integer part of $z$. This fact gives us the possibility to get stability results for (3.5) as a straightforward corollary of the corresponding stability results for FDEs. This argument goes back at least up to the paper [16] by Győri (see also $[8,18]$ ). For a detailed use of this technique, see the interesting paper [17] of Győri and Hartung, where the authors get new sufficient conditions for the 
asymptotic stability of linear difference equations. More recently, other stability conditions for nonlinear difference equations with applications in population dynamics were found using the same approach in [38,51].

In this section, we state a new result that can be proved using the EPCA approach, discuss some other stability results of the 3/2-type for difference equations that involve the discretized versions of Yorke-type conditions (3.2)-(3.4) and suggest some open problems.

For other stability results for difference equations related to the $3 / 2$ conditions, see the recent papers of Muroya et al. $[43,44]$ and their references.

\subsection{The sublinear case}

Let us consider the difference equation (3.5). The following hypothesis is a discretized version of condition (3.2):

(A1) There exist constants $\alpha_{n}>0$ such that, for every $\left(x_{0}, \ldots, x_{k}\right) \in \mathbb{R}^{k+1}$ and $n \in \mathbb{N}$,

$$
\alpha_{n} \min \left\{0, x_{0}, \ldots, x_{k}\right\} \leq f_{n}\left(x_{0}, \ldots, x_{k}\right) \leq \alpha_{n} \max \left\{0, x_{0}, \ldots, x_{k}\right\}
$$

We note that condition (A1) is a sort of sublinearity condition combined with a positive feedback condition. For example, if $f_{n}\left(x_{0}, \ldots, x_{k}\right) \equiv \alpha_{n} f(x)$, then (3.7) reads

$$
\min \{x, 0\} \leq f(x) \leq \max \{x, 0\}, \quad \forall x \in \mathbb{R},
$$

which is equivalent to $x f(x) \geq 0$ and $|f(x)| \leq|x|$ for all $x \in \mathbb{R}$.

As far as we know, condition (A1) was used for the first time in the frame of the stability theory for difference equations by Zhou and Zhang in [58], to prove the following result:

TheOREM 3.1 [58, TheOREM 1.3]. Assume that condition (A1) holds, and

$$
\limsup _{m \rightarrow \infty} \sum_{i=m}^{m+k} \alpha_{i}<\frac{3}{2}+\frac{1}{2(k+1)} .
$$

Then, every oscillatory solution of equation (3.5) tends to zero as $n \rightarrow \infty$.

An easy consequence of Theorem 3.1 is the following.

Corollary 3.2. Assume that (A1), (3.8), and the following condition hold:

(A2) If $\left\{x_{n}\right\}_{n \geq-k}$ is a sequence of real numbers such that $\lim _{n \rightarrow \infty} x_{n}=x^{*} \neq 0$, then the series $\sum_{n=0}^{\infty} f_{n}\left(x_{n}, \ldots, x_{n-k}\right)$ diverges.

Then, the zero solution of equation (3.5) is globally attracting.

Note that condition (A2) ensures that zero is the unique equilibrium of (3.5). This condition is used to prove that all non-oscillatory solutions of (3.5) converge to zero.

Remark 7. Condition (3.8) was established for the first time by Erbe, Xia and Yu [13] for a linear difference equation, namely, when $f_{n}\left(x_{n}, \ldots, x_{n-k}\right)=p_{n} x_{n-k}$ in equation (3.5), 
$p_{n} \geq 0, \quad \forall n \geq 0$. The authors of that paper were also motivated by the $3 / 2$-stability results obtained by Yorke and Yoneyama for FDEs.

Tkachenko and Trofimchuk [52] proved that condition (3.8) is sharp within the class of equations (3.5) with $f_{n}$ satisfying (A1). Assuming that (3.7) holds with $\alpha_{n}=\alpha$ for all $n \in \mathbb{N}$, Nenya, Tkachenko and Trofimchuk proved that the conclusions of Corollary 3.2 are still true replacing (3.8) by a weaker condition (see [45]); moreover, such a condition is sharp in the class of equations (3.5) satisfying

$$
\alpha \min \left\{0, x_{0}, \ldots, x_{k}\right\} \leq f_{n}\left(x_{0}, \ldots, x_{k}\right) \leq \alpha \max \left\{0, x_{0}, \ldots, x_{k}\right\}, \quad n \geq 0,
$$

for a positive constant $\alpha$ and all $\left(x_{0}, \ldots, x_{k}\right) \in \mathbb{R}^{k+1}$.

In our next result, we replace (A1) by a discrete version of Krisztin's condition (3.3) in order to get a new sufficient condition for the uniform asymptotic stability of (3.5). It can be easily proved by considering the EPCA (3.6) associated with (3.5), and applying the 3/2-type Theorem 1.1 in Krisztin's paper [27] (we leave the details to the reader).

THEOREM 3.3. Assume that there exist constants $\alpha_{0}, \ldots, \alpha_{k} \geq 0$ such that, for every $n \in \mathbb{N}$ and $\left(x_{0}, \ldots, x_{k}\right) \in \mathbb{R}^{k+1}$,

$$
\sum_{i=0}^{k} \alpha_{i} \min \left\{x_{0}, \ldots, x_{i}\right\} \leq f_{n}\left(x_{0}, \ldots, x_{k}\right) \leq \sum_{i=0}^{k} \alpha_{i} \max \left\{x_{0}, \ldots, x_{i}\right\} .
$$

Then, the zero solution of equation (3.5) is uniformly asymptotically stable if the following condition holds:

$$
0<\sum_{i=0}^{k}(i+1) \alpha_{i}<\frac{3}{2}
$$

For the linear equation with constant coefficients,

$$
x_{n+1}-x_{n}=-\sum_{i=0}^{k} a_{i} x_{n-i}, \quad n \geq 0 .
$$

Theorem 3.3 provides the following result.

COROLlary 3.4. Assume that $a_{i} \geq 0$ for all $i=0,1, \ldots, k$, and

$$
0<\sum_{i=0}^{k}(i+1) a_{i}<\frac{3}{2} .
$$

Then, the zero solution of (3.12) is exponentially stable.

Remark 8. The result of Corollary 3.4 has been recently improved by Kipnis and Komissarova [22]; they show that constant $3 / 2$ in condition (3.13) may be replaced by $\pi / 2$. Moreover, constant $\pi / 2$ is sharp. A different improvement of Corollary 3.4 can be found in the recent paper of Tang and Jiang [50]. Remarkably, this latest result was also motivated by Krisztin's paper [27]. 
The previous remark suggests the following question:

Open problem 1. Investigate whether it is true or not that the conclusions of Theorem 3.3 hold when constant $3 / 2$ in (3.11) is replaced by $\pi / 2$.

Motivated by condition (3.8) in Theorem 3.1, we also think it is interesting to study the following problem:

Open problem 2. Investigate if the conclusions of Theorem 3.3 hold when condition (3.11) is replaced by

$$
0<\sum_{i=0}^{k}(i+1) \alpha_{i}<\frac{3}{2}+\frac{1}{2(k+1)}
$$

Note that both problems are independent. Actually, $\pi / 2<3 / 2+1 /(2 k+2)$, only for $k=0,1, \ldots, 6$.

We underline the fact that not only linear equations fall in the scope of Theorem 3.3. It applies also to some nonlinear difference equations; in particular, those involving 'min-max' terms. For examples of such equations, see [9,25,35].

Example 3.5. Consider the following difference equation:

$$
x_{n+1}-x_{n}+p x_{n-1}+q \max \left\{x_{n}, x_{n-1}, x_{n-2}\right\}=0, n \geq 0,
$$

where $p, q$ are positive constants.

Note that it is a particular case of equation (2.18) considered in Section 2, with $m=1$. Thus, Propositions 2.7-2.9, based on the Halanay approach, apply to provide the following conditions for the exponential stability of the zero solution in (3.14).

Proposition 3.6. Any one of the following conditions ensures the global exponential stability of the zero solution in (3.14):

(1) $0<q<p<\frac{4}{9}$

(2) $p<\frac{1}{2}$ and $0<q<\frac{p(1-2 p)}{1+2 p}$ and

(3) $0<p+q<\frac{1}{2}$.

The application of Corollary 3.2 provides the condition $0<p+q<5 / 9$, which is sharper than condition (3) in Proposition 3.6. Using [45, Theorem 4], this condition is improved up to

$$
0<p+q<2-\sqrt{2} \approx 0.5857
$$

Theorem 3.3 also applies to this example, and it ensures that the zero solution in equation (3.14) is asymptotically stable if

$$
0<2 p+3 q<\frac{3}{2}
$$

One can check that (3.16) improves $(3.15)$ if $q<(4 \sqrt{2}-5) / 2 \approx 0.328$. 
We note that condition (1) in Proposition 3.6 ensures the global exponential stability of (3.14) in some cases, where both (3.15) and (3.16) fail. For example, if $p=0.4$ and $q \in(0.25,0.4)$.

\subsection{When (A1) fails}

In many important cases, function $f$ does not satisfy the sublinearity condition (A1). As an example, consider the discrete model for population dynamics

$$
N_{n+1}=N_{n} F\left(N_{n-k}\right) \text {, }
$$

where $N_{n}>0$ is the size of a population after $n$ generations, and it is assumed that the density-dependent mechanisms operate with a delay of $k$ generations (see [29]). Assuming that $F:(0, \infty) \rightarrow(0, \infty)$, and the initial data $N_{0}, \ldots, N_{-k}$ are positive, then $N_{n}>0$ for all $n \geq 0$. We suppose that there is a unique positive equilibrium $N^{*}$, which is defined by the relation $F\left(N^{*}\right)=1$. We say that $N^{*}$ is globally attracting if all positive solutions of (3.17) converge to $N^{*}$ as $n$ tends to infinity. In order to apply our results to equation (3.17), we make the change of variables $x_{n}=\ln \left(N^{*}\right)-\ln \left(N_{n}\right)$, which transforms the previous equation into

$$
x_{n+1}-x_{n}=-f\left(x_{n-k}\right) \text {, }
$$

with $f(x)=\ln \left(F\left(N^{*} e^{-x}\right)\right)$, for all $x \in \mathbb{R}$. Note that $N^{*}$ is globally attracting for (3.17) if and only if the zero solution of (3.18) is globally attracting in the sense of Definition 1.1.

A typical example of (3.17) is the Ricker difference equation with delay

$$
N_{n+1}=N_{n} e^{r\left(1-N_{n-k}\right)},
$$

where $r$ is a positive constant. Thus, $N^{*}=1$, and (3.19) takes the form (3.18) with $f(x)=r\left(1-e^{-x}\right)$. Clearly, $f$ does not satisfy the sublinearity condition $|f(x)| \leq \alpha|x|$ for any $\alpha<0$, and hence Corollary 3.2 does not apply.

Next, we state a generalization of Corollary 3.2 obtained by Tkachenko and Trofimchuk [52] under an assumption weaker than (A1), allowing the case $f(x)=r\left(1-e^{-x}\right)$. First, we introduce two new assumptions:

$\left(\mathrm{A} 1^{\prime}\right) \quad$ There exist constants $\alpha_{n}>0$ and $\beta>0$ such that, for every $n \in \mathbb{N}$,

$$
\alpha_{n} R\left(\min \left\{0, x_{0}, \ldots, x_{k}\right\}\right) \leq f_{n}\left(x_{0}, \ldots, x_{k}\right) \leq \alpha_{n} R\left(\max \left\{0, x_{0}, \ldots, x_{k}\right\}\right),
$$

where $R(x)=x /(1+\beta x)$, the first inequality holds for all $\left(x_{0}, \ldots, x_{k}\right) \in \mathbb{R}^{k+1}$, and the second one only for those $\left(x_{0}, \ldots, x_{k}\right) \in \mathbb{R}^{k+1}$ such that $\min \left\{x_{i}: 0 \leq i \leq k\right\}>-1 / \beta$. (A3) There exists $\vartheta: \mathbb{R} \rightarrow \mathbb{R}_{+}$such that $f_{n}\left(x_{0}, \ldots, x_{k}\right) \leq \vartheta(s)$ for every $\left(x_{0}, \ldots, x_{k}\right) \in \mathbb{R}^{k+1}$ with $\min \left\{x_{i}: 0 \leq i \leq k\right\} \geq s$.

Note that condition $\left(\mathrm{A}^{\prime}\right)$ is the discrete version of the generalized Yorke condition (3.4). It was used for the first time in [38].

TheOrem 3.7 [52, TheOrem 1.3]. Assume that the hypotheses $\left(A 1^{\prime}\right),(A 2)$ and $(A 3)$ are satisfied. If condition (3.8) holds, then the zero solution of equation (3.5) is globally attracting. 
Using the following corollary of Theorem 3.7 (that improves the result of [38, Corollary 4.3]), it is easy to prove that the equilibrium $N^{*}$ in the delayed Ricker equation (3.19) is globally attracting if

$$
r \leq \frac{3}{2(k+1)}+\frac{1}{2(k+1)^{2}}
$$

COROLlary 3.8. Assume that $\mathrm{f}$ satisfies the following conditions:

(1) $f(0)=0, f^{\prime \prime}(0) \neq 0, f^{\prime}(x)>0$ for all $x \in \mathbb{R}$, and $f$ is bounded from above.

(2) $f \in C^{3}(\mathbb{R}, \mathbb{R})$, and $(S f)(x)<0$ for all $x \in \mathbb{R}$, where

$$
(S f)(x)=\frac{f^{\prime \prime \prime}(x)}{f^{\prime}(x)}-\frac{3}{2}\left(\frac{f^{\prime \prime}(x)}{f^{\prime}(x)}\right)^{2}
$$

is the Schwarzian derivative of $f$.

Then, the zero solution of equation (3.18) is globally stable if

$$
f^{\prime}(0) \leq \frac{3}{2(k+1)}+\frac{1}{2(k+1)^{2}} \text {. }
$$

Remark 9. It is immediate to check that conditions (1) and (2) hold for $f(x)=r\left(1-e^{-x}\right)$. In particular, $(S f)(x)=-1 / 2$ for all $x \in \mathbb{R}$.

For $k=0$, Corollary 3.8 was proved in [32]. In this case, the conclusion may be written in the following way.

PROPOSITION 3.9. Consider the first-order difference equation

$$
x_{n+1}-x_{n}=-f\left(x_{n}\right) \text {. }
$$

Assume that f satisfies conditions (1) and (2) in the statement of Corollary 3.8. Then, the local asymptotic stability of the zero solution of (3.21) implies its global asymptotic stability.

See $[7,32,33]$ for the application of this result to several population models.

For equation (3.19) and other forms of (3.17), Levin and May affirmed in [29] that '... extensive numerical studies suggest that the local asymptotic stability imply global stability in those models'. Corollary 3.8 is a good support for this conjecture, since the curves defined by the global stability condition (3.20) and the condition for the local asymptotic stability $f^{\prime}(0)<2 \cos ((k \pi) /(2 k+1)$ ) given in [29] are very close (see [38], for more details). This motivates the following conjecture.

Conjecture 3.10 [38, Conjecture 4.8]. Assume that $f$ satisfies conditions (1) and (2) from the statement of Corollary 3.8. Then, the zero solution of equation (3.18) is globally stable whenever it is asymptotically stable.

As an interesting challenge, in order to complement the result of Corollary 3.8 and support a positive answer to the previous conjecture, we propose the study of the following question.

Open problem 3. Investigate if the conclusions of Corollary 3.8 hold when condition (3.20) is replaced by

$$
f^{\prime}(0) \leq \frac{\pi}{2(k+1)}
$$




\section{Acknowledgements}

This research was supported in part by the Spanish Ministry of Science and Innovation and FEDER, grant MTM2007-60679.

The author is thankful to an anonymous referee for his/her useful remarks.

\section{References}

[1] R.P. Agarwal, Difference equations and Inequalities. Theory, Methods, and Applications, 228, 2nd ed., Monographs and Textbooks in Pure and Applied Mathematics, Marcel Dekker, Inc., New York, 2000.

[2] R.P. Agarwal, Y. Kim, and S.K. Sen, New discrete Halanay inequalities: Stability of difference equations, Commun. Appl. Anal. 12 (2008), pp. 83-90.

[3] C.T.H. Baker and E. Buckwar, Exponential stability in p-th mean of solutions, and of convergent Euler-type solutions, of stochastic delay differential equations, J. Comput. Appl. Math. 184 (2005), pp. 404-427.

[4] C.T.H. Baker and A. Tang, Generalized Halanay inequalities for Volterra functional differential equations and discretized versions Numerical analysis report, University of Manchester, (1996). Available at http://www.maths.man.ac.uk/nareports/narep299.ps.gz

[5] N.S. Bay and V.N. Phat, Stability analysis of nonlinear retarded difference equations in Banach spaces, Comput. Math. Appl. 45 (2003), pp. 951-960.

[6] L. Berezansky, E. Braverman, and E. E. Liz, Sufficient conditions for the global exponential stability of nonautonomous higher order difference equations, J. Differ. Equat. Appl. 11 (2005), pp. 785-798.

[7] E. Braverman and S.H. Saker, Periodic solutions and global attractivity of a discrete delay host macroparasite model, J. Differ. Equat. Appl. 16 (2010), pp. 789-806.

[8] K. Cooke, I. Gyôri, Numerical approximation of the solutions of delay differential equations on an infinite interval using piecewise constant arguments, Comput. Math. Appl. 28 (1994), pp. 81-92.

[9] M. Csörnyei, M. Laczkovich, Some periodic and non-periodic recursions, Monatsh. Math. 132 (2001), pp. 215-236.

[10] P. Cull, M. Flahive, and R. Robson, Difference equations. From rabbits to chaos, Springer, New York, 2005.

[11] S. Elaydi, An introduction to difference equations, 3rd ed., Springer Verlag, New York, 2005.

[12] H.A. El-Morshedy, New explicit global asymptotic stability criteria for higher order difference equations, J. Math. Anal. Appl. 336 (2007), pp. 262-276.

[13] L.H. Erbe, H. Xia, and S. Yu, Global stability of a linear nonautonomous delay difference equation, J. Differ. Equat. Appl. 1 (1995), pp. 151-161.

[14] K. Ey, C. Pötzsche, Asymptotic behavior of recursions via fixed point theory, J. Math. Anal. Appl. 337 (2008), pp. 1125-1141.

[15] G. Gandolfo, Economic dynamics: methods and models, 16, 2nd ed., Advanced Textbooks in Economics, North-Holland, Amsterdam/New York, 1980.

[16] I. Györi, On approximation of the solutions of delay differential equations by using piecewise constant arguments, Int. J. Math. Math. Sci. 14 (1991), pp. 111-126.

[17] I. Győri and F. Hartung, Stability in delay perturbed differential and difference equations, in Topics in functional differential and difference equations, T. Faria and P. Freitas, eds., 29, Fields Inst. Commun, Amer. Math. Soc., Providence, RI, 2001, pp. 181-194.

[18] I. Győri and M. Pituk, Comparison theorems and asymptotic equilibrium for delay differential and difference equations, Dynam. Syst. Appl. 5 (1996), pp. 277-302.

[19] A. Halanay, Differential equations: Stability, oscillations, time lags, Academic Press, New York, 1966.

[20] A. Ivanov, E. Liz, and S. Trofimchuk, Halanay inequality, Yorke 3/2 stability criterion, and differential equations with maxima, Tohoku Math. J. 54 (2002), pp. 277-295.

[21] M. Kipnis and D. Komissarova, Stability of a delay difference system, Adv. Difference Equ. Art. ID 31409 (2006), p. 9.

[22] M. Kipnis, D. Komissarova, A note on explicit stability conditions for autonomous higher order difference equations, J. Difference Equ. Appl. 13 (2007), pp. 457-461.

[23] P.E. Kloeden and A.M. Rubinov, A generalization of the Perron-Frobenius theorem, Nonlinear Anal. 41 (2000), pp. 97-115. 
[24] V.L. Kocić and G. Ladas, Global behavior of nonlinear difference equations of higher order with applications, 256, Mathematics and its Applications, Kluwer Academic Publishers Group, Dordrecht, 1993.

[25] U. Krause, The asymptotic behavior of monotone difference equations of higher order, Comput. Math. Appl. 42 (2001), pp. 647-654.

[26] U. Krause and M. Pituk, Boundedness and stability for higher order difference equations, J. Differ. Equat Appl. 10 (2004), pp. 343-356.

[27] T. Krisztin, On stability properties for one-dimensional functional differential equations, Funkcial. Ekvac. 34 (1991), pp. 241-256.

[28] A.Y.T. Leung, J.N. Xu, and S. Tsui, Nonlinear delay difference equations for housing dynamics assuming heterogeneous backward-looking expectations, Appl. Math. Mech. (English Ed.) 28 (2007), pp. 785-798.

[29] S.A. Levin, R. May, A note on difference delay equations, Theor. Pop. Biol. 9 (1976), pp. $178-187$.

[30] B.Y. Liu and H. Gui, Robust output feedback control for uncertain discrete systems with time delays, Acta Automat. Sinica 31 (2005), pp. 804-807.

[31] E. Liz, On explicit conditions for the asymptotic stability of linear higher order difference equations, J. Math. Anal. Appl. 303 (2005), pp. 492-498.

[32] E. Liz, Local stability implies global stability in some one-dimensional discrete single-species models, Discrete Contin. Dyn. Syst., Ser. B 7 (2007), pp. 191-199.

[33] E. Liz, A sharp global stability result for a discrete population model, J. Math. Anal. Appl. 330 (2007), pp. 740-743.

[34] E. Liz and B. Ferreiro, A note on the global stability of generalized difference equations, Appl. Math. Lett. 15 (2002), pp. 655-659.

[35] E. Liz, A. Ivanov, and J.B. Ferreiro, Discrete Halanay-type inequalities and applications, Nonlinear Anal. 55 (2003), pp. 669-678.

[36] E. Liz and M. Pituk, Asymptotic estimates and exponential stability for higher order monotone difference equations, Adv. Difference Equ. (2005), pp. 41-55.

[37] E. Liz, V. Tkachenko, and S. Trofimchuk, Yorke and Wright 3/2-stability theorems from a unified point of view, Discrete Contin. Dynam. Syst. (2003), pp. 580-589.

[38] E. Liz, V. Tkachenko, and S. Trofimchuk, Global stability in discrete population models with delayed-density dependence, Math. Biosci. 199 (2006), pp. 26-37.

[39] R. Memarbashi, Sufficient conditions for the exponential stability of nonautonomous difference equations, Appl. Math. Lett. 21 (2008), pp. 232-235.

[40] R. Memarbashi, On the stability of nonautonomous difference equations, J. Difference Equ. Appl. 14 (2008), pp. 301-307.

[41] S. Mohamad and K. Gopalsamy, Continuous and discrete Halanay-type inequalities, Bull. Aus. Math. Soc. 61 (2000), pp. 371-385.

[42] S. Mohamad and K. Gopalsamy, Exponential stability of continuous-time and discrete-time cellular neural networks with delays, Appl. Math. Comput. 135 (2003), pp. 17-38.

[43] Y. Muroya, E. Ishiwata and N. Guglielmi, Global stability for nonlinear difference equations with variable coefficients, J. Math. Anal. Appl. 334 (2007), pp. 232-247.

[44] Y. Muroya, E. Ishiwata, and N. Guglielmi, New global stability conditions for a class of difference equations, Front. Math. China 4 (2009), pp. 131-154.

[45] O. Nenya, V. Tkachenko and S. Trofimchuk, On sharp conditions for the global stability of a difference equation satisfying the Yorke condition, Ukrainian Math. J. 60 (2008), pp. 78-90.

[46] Z. Qiang, More relaxed condition for dynamics of discrete time delayed Hopfield neural networks, Chin. Phys. B 17 (2008), pp. 125-128.

[47] H. Sedaghat, Geometric stability conditions for higher order difference equations, J. Math. Anal. Appl. 224 (1998), pp. 255-272.

[48] H. Sedaghat, Nonlinear difference equations. Theory with applications to social science models., Mathematical Modelling: Theory and Applications, Kluwer Academic Publishers, Dordrecht, 2003, p. 15.

[49] S. Stević, Behavior of the positive solutions of the generalized Beddington-Holt equation, Panamer. Math. J. 10 (2000), pp. 77-85.

[50] X.H. Tang and Z. Jiang, Asymptotic behavior of Volterra difference equation, J. Difference Equ. Appl. 13 (2007), pp. 25-40. 
[51] V. Tkachenko and S. Trofimchuk, Global stability in difference equations satisfying the generalized Yorke condition, J. Math. Anal. Appl. 303 (2005), pp. 173-187.

[52] V. Tkachenko and S. Trofimchuk, global attractivity criterion for nonlinear non-autonomous difference equations, J. Math. Anal. Appl. 322 (2006), pp. 901-912.

[53] S. Udpin and P. Niamsup, New discrete type inequalities and global stability of nonlinear difference equations, Appl. Math. Lett. 22 (2009), pp. 856-859.

[54] T. Yoneyama, On the 3/2 stability theorem for one-dimensional delay-differential equations, J. Math. Anal. Appl. 125 (1987), pp. 161-173.

[55] J.A. Yorke, Asymptotic stability for one dimensional differential-delay equations, J. Differential Equat. 7 (1970), pp. 189-202.

[56] Z. Zeng and J. Wang, Multiperiodicity of discrete-time delayed neural networks evoked by periodic external inputs, IEEE Trans. Neural Netw. 17 (2006), pp. 1141-1151.

[57] Q. Zhang, X. Wei, and J. Xu, global exponential stability of discrete-time Hopfield neural networks with variable delays, Art. ID 67675 Discrete Dyn. Nat. Soc. (2007), p. 9.

[58] Z. Zhou and Q. Zhang, Uniform stability of nonlinear difference systems, J. Math. Anal. Appl. 225 (1998), pp. 486-500. 